\title{
Nanobodies for isoform-specific study of voltage-gated sodium channels (Navs)
}

\author{
L Srinivasan ${ }^{1}$, V Alzogaray ${ }^{2}$, D Selvakumar ${ }^{3}$, J Yoder ${ }^{1}$, K. M. Wright ${ }^{1}$, S Klinke ${ }^{2}$, F A. Goldbaum ${ }^{2}$, L \\ Mario Amzel ${ }^{1,3}$, S B Gabelli ${ }^{1,4,5}$ \\ ${ }^{1}$ Department of Biophysics and Biophysical Chemistry, The Johns Hopkins School of Medicine, \\ Baltimore, USA, ${ }^{2}$ Fundacion Instituto Leloir, Buenos Aires, Argentina, ${ }^{3}$ ForteBio, Fremont, CA, \\ USA, ${ }^{4}$ Department of Medicine, The Johns Hopkins University School of Medicine, Baltimore, USA, \\ ${ }^{5}$ Department of Oncology, The Johns Hopkins University School of Medicine, Baltimore, USA \\ Isriniv2@jhmi.edu
}

Nav1.4 and Nav1.5 are voltage-gated sodium channels that play an important role in the generation of action potential in excitable tissues. Navs control the passage of sodium ions into cells in response to changes in cellular membrane potential. Myotonia, hyperkalemic periodic paralysis, LongQT syndrome and Brugada syndrome are human genetic diseases caused by dysfunction of Nav proteins that harbor mutations in their C-terminal (CT), cytoplasmic regions. To study the Navs proteins with isoform-specificity, we have selected nanobodies; single, variable heavy-chain only antibody domains ( $\mathrm{VHH}$ domains, 15kDa) derived from llamas. We generated a nanobody phage library by immunizing a llama with purified CTNav1.4 in complex with Calmodulin (CTNav1.4$\mathrm{CaM}$ ). High-affinity nanobody clones to CTNav1.4-CaM complex were selected by immune panning from the phage library and used for periplasmic expression in E. coli. Two anti-CTNav1.4-CaM nanobodies; Nb17 and $\mathrm{Nb} 82$ were expressed and purified to homogeneity, while $\mathrm{Nb} 82$ was further crystallized and structure determined to $2.0 \AA$ resolution. The asymmetric unit includes four copies with almost identical conformations. The nanobodyfold is decorated by two $\Pi$-helices present in CDR1 and CDR3, between $\beta 3-\beta 4$ and $\beta 9-\beta 10$ loops. CDR3, the major contributor for antigen recognition and specificity, folds as a random coil that wraps around the $\beta$-sheets $\beta 6-\beta 5-\beta 4-$ $\beta 9$ finishing up in a П-helix of seven residues. Interestingly, Nb82 stabilizes the CTNav1.4-CaM complex as observed by a 'right-shift' of $13^{\circ} \mathrm{C}$ in the melt curve of CTNav1.4-CaM, while forming a stable CTNav1.4$\mathrm{CaM}+\mathrm{Nb}$ complex on size exclusion chromatography.

Acta Cryst. (2020). A76, a59 